\title{
TP53 and EGFR Mutational Status in Thymoma: A Genetic Sequencing Study
}

\author{
Elisna Syahruddin ${ }^{1,2}$, Jamal Zaini ${ }^{1 *}$, Ruth Sembiring ${ }^{3}$, Romy Baginta ${ }^{3}$, Muhamad \\ Rizqy Fadhillah ${ }^{1}$, Dimas Ramadhian Noor ${ }^{2}$
}

\begin{abstract}
Background and objective: Thymoma is a rare malignant tumor that usually with an indolent presentation, which was falsely assumed to be benign previously. The tumor suppressor P53 (TP53) and EGFR gene mutate most frequently in human cancers. We tried to investigate the presence of TP53 and EGFR mutations among thymoma patients referred to an Indonesian referral respiratory hospital and to discuss its potential role in thymoma management and prognosis. Material and methods: Surgically resected tumor tissues were collected from thymoma patients and then underwent genomic analysis. PCR was performed on the extracted Paraffinized DNA to amplify exon 6 of TP53 and exons 18, 19, and 21 of $E G F R$. The evaluation of mutational status was done using direct sequencing and sequence analysis of purified PCR products. Results: Among 27 collected samples, TP53 exon 6 mutation, namely missense mutation and nonsense mutation, was observed in two samples (7.4\%). EGFR exon 18 mutation, namely $E 709 \mathrm{~K}$ and nonsense mutation, was found in 2 samples (7.4\%). An intronic mutation in EGFR exon 19 (3.7\%) and exon 21 (3.7\%) was observed in one sample. Conclusion: TP53 and EGFR mutations were not most frequent, so it seems that these genes are not involved in the pathogenesis of thymoma in Indonesian patients. Nevertheless, we found two samples with a significant mutation in $p 53$ and EGFR genes, suggesting further research on thymoma prognostification and targeted therapy.
\end{abstract}

Keywords: EGFR-TP53- Thymoma

Asian Pac J Cancer Prev, 23 (1), 109-114

\section{Introduction}

Thymoma is a rare malignant tumor usually with an indolent presentation, which was falsely assumed to be benign previously. The actual incidence of thymoma is unknown; however, $47 \%$ of anterior mediastinum tumors are thymoma (Detterback et al., 2013). From 1970 to 1990, thymoma accounted for $24 \%$ of 137 cases of surgically resected mediastinum tumor in Persahabatan National Respiratory Referral Hospital Indonesia (PDPI, 2003). Around one-third of thymoma patients suffer from myasthenia gravis (Detterback et al., 2013).

Thymoma staging, histologic type, and complete eradication through any treatment modalities are significant to increase the survival of patients and reduce its recurrence rate. Prognosis of thymoma is worse at higher stages of the disease. The patients at the advanced stage of the thymoma have a 5-year median survival of $69 \%$. Currently, the treatment choice in thymoma is surgery providing a better prognosis (Detterback et al., 2013, NCCN, 2016). However, the role of molecular therapy in this tumor, such as targeted therapy, is unknown.

The tumor suppressor 53 (TP53) is the most frequently mutated gene in human cancer and known as "the guardian of the genome". TP53 role is a gatekeeper of cell growth and division acting a central role in gene induction for cell cycle arrest and apoptosis when DNA is damaged (Giacomelli et al., 2018). Thus, this protein role is vital for suppressing the growth of the tumor and reducing cell malignant transformation. Alteration of this gene was observed in somatic mutation with a specific clinical phenotype for each cancer. TP53 is known as a marker that can predict therapeutic response and prognosis in squamous cell lung carcinoma (Feng $\mathrm{Xu}$ et al., 2020, Kobayashi et al., 2015). In addition, its higher level detection was associated with better prognosis in other cancers, namely, acute myeloid leukemia (Sadek, 2020).

Epidermal growth factor receptor (EGFR) is a receptor with tyrosine kinase activity, which is important in transducing signals from extracellular into the intracellular (De Luca, 2008). EGFR mutation has been reported in non-small cell lung carcinoma cases and is frequently

${ }^{1}$ Department of Pulmonology and Respiratory Medicine, Faculty of Medicine, Universitas Indonesia/Persahabatan National Respiratory Referral Hospital, Jakarta, Indonesia. ${ }^{2}$ Human Cancer Research Centre-IMERI-Faculty of Medicine Universitas Indonesia. ${ }^{3}$ Pathology Unit, Persahabatan National Respiratory Referral Hospital, Jakarta, Indonesia. *For Correspondence: jamal.zaini@gmail.com 
mutated in exon 18, 19, 20, and 21. Commonly, the mutation is missense. For instance, G719S, L858R, and L861Q (Lynch et al., 2004, Kobayashi et al., 2015) mutations are characterized by the short deletion in exon 19 and point mutations in exons 19 and 21. Those three mutations are associated with higher sensitivity to tyrosine kinase inhibitors (TKI). However, D761Y (Balak et al.,2006) and T790M (Lee et al., 2021) mutations are resistant to this treatment. The presence of EGFR mutation also better predicts overall survival (OS) (Okamoto et al., 2018), response to TKI therapy as well as progression-free survival ( $>9$ months) in NSCLC (Pas-Arez et al., 2009, Lynch et al. 2004).

This study investigated the presence of TP53 and $E G F R$ mutations among thymoma patients in an tertiary respiratory referral hospital, Indonesia, and discussed its impact with respect to prognostification and therapeutic aspects on anti-EGFR therapy. Genetic sequencing using direct sequencing and sequence analysis in TP53 exon 6 and EGFR exon 18, 19, and 21 were also done.

\section{Materials and Methods}

\section{Study participants and DNA extraction}

This study was approved by the Ethic Committee of Persahabatan Hospital (No 139/KEPK-RSUPP/12/2020). Thirty deparaffinized histopathology samples were collected from thymoma patients diagnosed at the Persahabatan National Refferal Respiratory Hospital, Indonesia. The samples were obtained from surgically resected tumor tissues and chosen to undergo genomic analysis. DNA was extracted from Formalin Fixed Paraffin Embedded using Quick-DNA FFPE mini prep (Zymo Research, USA) according to manufacturer's protocols as follows. Approximately, FFPE were sliced using microtome in 1-2 slices and $4 \mu \mathrm{m}$ thickness each. Slices were transferred to $1.5 \mathrm{ml}$ centrifuge tubes. Deparaffinization, tissue digestion, and DNA purifications were carried out according to manufacturer's protocols. The DNA was eluted in $50 \mu \mathrm{l}$ of DNA elution buffer. DNA concentrations and purity were measured using a Biodrop Nano spectrophotometer. Further, a polymerase chain reaction (PCR) was performed for all DNA samples. The clinicopathological data of specimens, including patient's age and sex, WHO subtype, and Masaoka staging were also collected.

\section{Mutation analysis}

PCR amplification was performed for all DNA samples for TP53 exon 6 and EGFR exon 18, 19, and 21 using MyTaq ${ }^{\mathrm{TM}}$ Hotstar RedMix PCR mix (Bioline, United Kingdom). The process was done based on the manufacturer's protocol. The primers and their product size base pair are presented in Table 1. A PCR reaction consisted of PCR buffer, 0,2 mM dNTP, $0.3 \mu \mathrm{M}$ forward and reverse primers, 1.25 Taq DNA polymerase, and 250 ng of DNA with a total volume of $50 \mu \mathrm{L}$. PCR cycling conditionsincluded pre-denaturation step in $95^{\circ} \mathrm{C}$ for 1 $\min$. The steps were followed by denaturation $95^{\circ} \mathrm{C}$ for 15 $\mathrm{s}$, annealing in $65^{\circ} \mathrm{C}$ for $15 \mathrm{~s}$, and extension $72^{\circ} \mathrm{C}$ in $15 \mathrm{~s}$ for 40 cycles. The steps were continued by final extension under $72^{\circ} \mathrm{C}$ for 10 minutes afterward.

The PCR product was then loaded and analyzed using $2 \%$ of agarose gel electrophoresis. Non-template control was done using PCR primer mix without any DNA to detect unwanted DNA contaminations within PCR mix. Samples were then run in $2 \%$ of agarose electrophoresis in Tris Buffer EDTA pH 8.0.

\section{DNA sequencing and sequence analysis}

DNA sequencing was performed by using Sanger Sequencing according to 1 st BASE Asia standard Laboratory procedure (First base Asia, Malaysia). The sequencing results were imported, converted to reverse complement, and analyzed using ClustalW in BioEdit Sequence Alignment Editor 7.2.5 editions. The reverse sequence was also analyzed to human reference genome NG_007524.2 (LRG_433) using nBLAST NCBI and standard preferences. The BLASTn results were also aligned with corresponding coding sequences (CDS). Lastly, the sequence was analyzed using FinchTv 1.4 and reverse complement views.

\section{Results}

\section{Tumor characteristics}

Clinical characteristics of the entire samples are present in Table 2. The majority of collected samples wereotained from male patients with a median age of 55 . The primary thymoma staging and WHO subtype was IIIB and type A, respectively.

Genomic DNA was extracted from 30 surgically resected thymoma tissues. Three samples were failed to amplify for TP53 exon 6 and EGFR exon 18, 19, and 21 so they were discarded. Twenty seven samples were successfully underwent PCR, gene sequencing, nBLAST, and electropherogram analysis.

\section{Detection of mutation}

We observed several mutations in different exons of our samples, and the findings are summarized in Table 3. TP53 mutations were detected in two samples. These samples showed different base pairs illustrating a point mutation. A missense mutation was observed in one sample, at positions 155 from $\mathrm{G}>\mathrm{A}$ in exon 6 . Another sample had substitution from $19319 \mathrm{~T}>\mathrm{G}$ (silent mutation),

Table 1. Primer Sequences

\begin{tabular}{lccc}
\hline Gene and Exon & Forward Primer & Reverse Primer & PCR Product Size (bp) \\
\hline TP53 Exon 6 & CAGGTCTCCCCAAGGCGCAC & GCAAGCAGAGGCTGGGGCAC & 219 \\
EGFR Exon 18 & ATGTCTGGCACTGCTTTCCA & CACAGGACCACTGATTACTG & 392 \\
EGFR Exon 19 & TCACTGGGCAGCATGTGGCA & CAGCTGCCAGACATGAGAAA & 241 \\
EGFR Exon 21 & ATTCGGATGCAGAGCTTCTT & CCTGGTGTCAGGAAAATGCT & 265 \\
\hline
\end{tabular}


EXON 6 missense mutation (18319 A $>$ G)

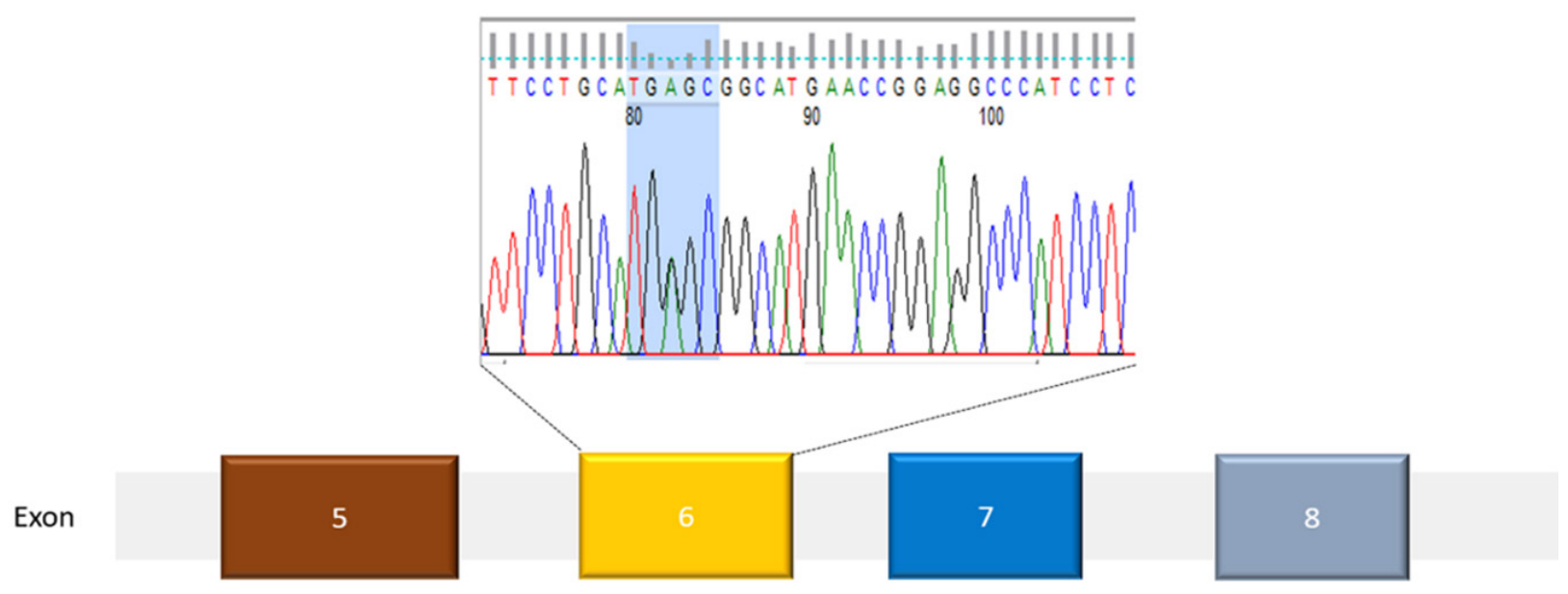

Figure 1. Amino Acid and Nucleotide Sequence Changes in Exon 6 Missense in TP53 Gene

Table 2. Clinicopathological Characteristic of Thymoma Cancer Discuss in This Study

\begin{tabular}{|c|c|}
\hline Clinicopathological Features & $\mathrm{n}(\%), \mathrm{N}=27$ \\
\hline \multicolumn{2}{|l|}{ Sex } \\
\hline Male & $12(44.4)$ \\
\hline Female & $15(55.5)$ \\
\hline \multicolumn{2}{|l|}{ Age (Years) } \\
\hline Mean & 55 \\
\hline Range & $32-76$ \\
\hline \multicolumn{2}{|l|}{ WHO Subtype } \\
\hline $\mathrm{A}$ & $15(55.5)$ \\
\hline $\mathrm{AB}$ & $1(3.7)$ \\
\hline $\mathrm{B} 1$ & $11(40.7)$ \\
\hline $\mathrm{B} 2$ & $0(0)$ \\
\hline B3 & $0(0)$ \\
\hline \multicolumn{2}{|l|}{ Masaoka Staging } \\
\hline I & $0(0)$ \\
\hline IIA & $0(0)$ \\
\hline IIB & $0(0)$ \\
\hline IIIA & $2(7.4)$ \\
\hline IIIB & $15(55.5)$ \\
\hline IVA & $8(29.6)$ \\
\hline IVB & $2(7.4)$ \\
\hline
\end{tabular}

as confirmed by BLAST analysis and electrophoregram (Figure 1). Based on CDS, this mutation caused changes in the expression of serine to glycine amino acid. These results may indicate heterozygous mutations due to detections of both A and G, respectively.

Mutations in exon 19 occurred in two samples (c.2125 G>A, leading to substitution of E709K, missense mutation, and c.2076 T $>$ C, a nonsense mutation) (Figure 2 ). In exon 20 , no deletions occured in the most frequent locations, usually found in base 746-750 of EGFR. However, intronic substitution was located at g.160661 C $>$ A. Only EGFR exon 21 intronic substitution mutation
Table 3. Summary of EGFR and TP53 Mutation in Thymoma

\begin{tabular}{ll}
\hline Gene location & $\begin{array}{l}\text { Frequencies } \\
\text { of samples (\%) }\end{array}$ \\
\hline TP53 Exon 6 & $1 / 27(3.7 \%)$ \\
G155A & $1 / 27(3.7 \%)$ \\
$\quad$ Silent mutation & \\
EGFR Exon 18 & $1 / 27(3.7 \%)$ \\
$\quad$ E709K & $1 / 27(3.7 \%)$ \\
$\quad$ Nonsense mutation (c.2076T $>$ C) & \\
EGFR Exon 19 & $1 / 27(3.7 \%)$ \\
$\quad$ Intronic substitution (g.160661C $>\mathrm{A})$ & \\
EGFR Exon 21 & \\
$\quad$ Intronic substitution (g.177642C $>\mathrm{T})$ & $1 / 27(3.7 \%)$ \\
\hline
\end{tabular}

was found in one sample at g.177642 C $>\mathrm{T}$.

\section{Discussion}

Based on the findings of this study, there were several mutations, including missense and silent mutation, in TP53 exon 6 , and missense (E709K) mutation in EGFR exon 18 . In addition, it was found that two samples had intronic substitution in exons 20 and 21. In a previous study, p53 mutation was found in thymoma, but with lower frequencies or in zero prevalence (Franz et al., 2017, Oberndorfer et al., 2019, Peric et al., 2019, Girard et al., 2009, Lei Yu et al., 2019, Li et al., 2019). The aformentioned study showed that $7,14 \%$ of $p 53$ genes were mutated in the samples. In general, a missense mutation in p53 gene is the most common finding in human cancers (73.16\%) (Bouaoun et al., 2016), which is in line with our study.

P53 is known as the most commonly mutated gene in malignant tumors. Thus, p53 mutation could be a predictor of tumor aggressivity since its appearance is common in thymic carcinoma. Moreira et al., (2015) observed that not a single sample of B3 thymoma had p53 mutation; whereas, thymic carcinoma showed chromosomal 


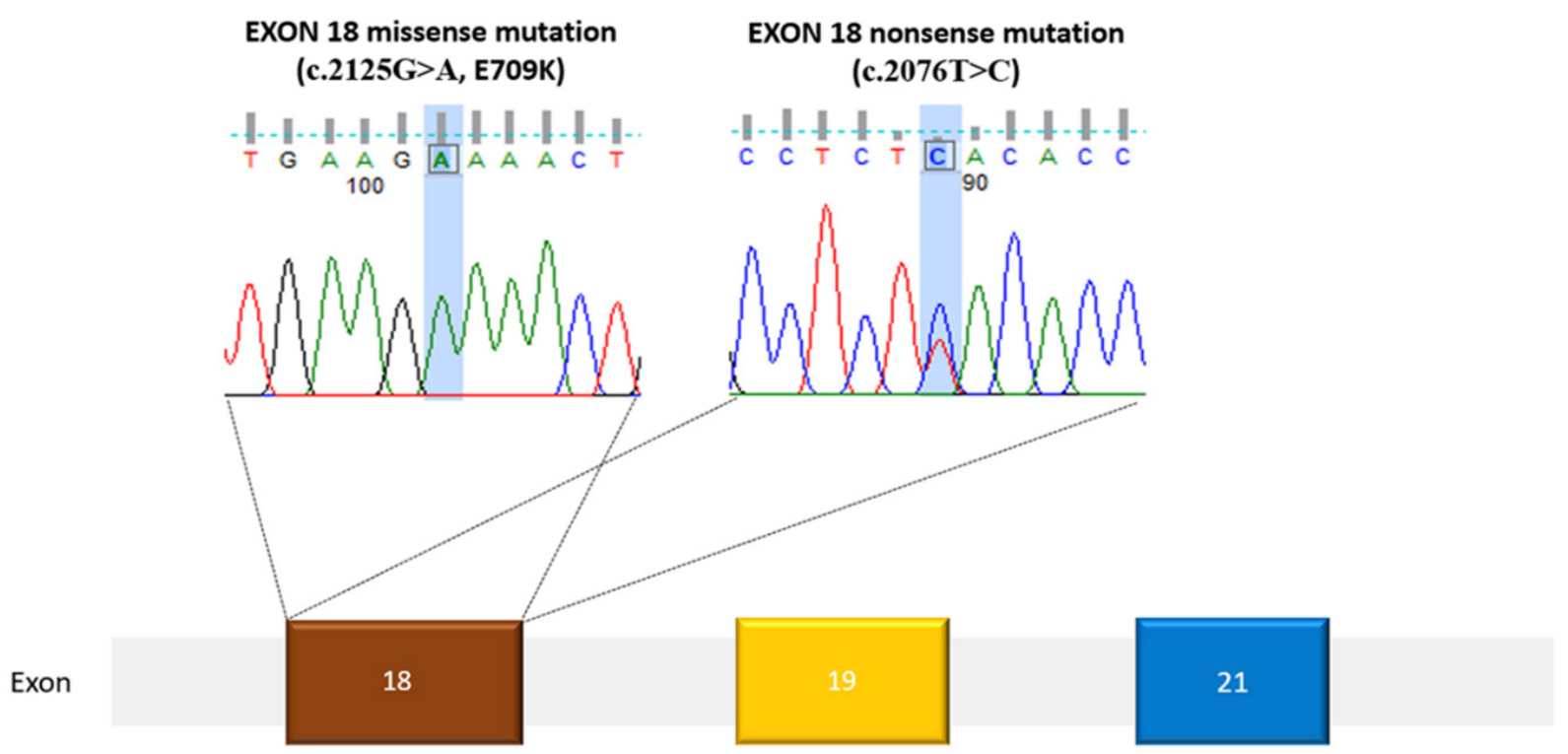

Figure 2. Amino Acid and Nucleotide Sequence Changes in Exon 18 Missense and Nonsense Mutation in EGFR Gene

gains and losses. Moreover, thymic carcinoma had p53 chromosomal gain or losses, which are responsible for its aggressive behaviour. Other recent studies also confirmed that p53 and EGFR were frequently spotted in thymic carcinoma (Sakane et al., 2021; Zhan et al., 2018; Prays et al., 2021).

Previous studies reported contrasting findings on the presence of EGFR mutation in thymoma . For instance, Suzuki et al., found that direct sequencing in did not reveal any EGFR missense mutation in 38 samples obtained from Japanese patients diagnosed with thymoma (Suzuki et al., 2006). On the other hand, Yoh et al., (2008) depict two of 20 patients with thymoma. They also observed that all of the EGFR mutations found were missense mutations in exon 21. Further, in another study, Sakane et al., performed direct sequencing in exon 19 of EGFR and indicated no mutation in EGFR gene (Sakane et al., 2019). Based on these studies and our results, it seems that EGFR mutation in thymoma is rare and potentially might not be related to thymoma carcinogenesis.

To best of our knowledge, few studies discussed intronic mutation in thymoma. The intronic mutation is also known as a cancer-related mutation, especially in mis-splicing more than 20 base pairs (Jung et al., 2021). However, Meister et al., (2007) reported that some thymoma patients had a single base pair variation in an intron and also a non-tumor cell. However, a recent meta-analysis reported that polymorphism in TP53 codon $72 \mathrm{G}>\mathrm{C}$ might be associated with increased risk of thyroid cancer (Nedooshan, 2016). Hence, further studies are needed to reveal our finding correlating with its clinical implication, disease progressivity, targeted therapy, and prognosis.

Since $p 53$ and EGFR are possibly not the founders of carcinogenesis in this tumor, another somatic mutation,-rather than p53 and EGFR, should be considered as the possible driven carcinogenesis gene in thymoma. Using a high-throughput approach, one study in Serbia revealed that ten genes other than EGFR, including FBXW7, FGFR3, GNAQ, GNA11, HNF1A, KIT, $M E T$, PIK3CA, PTEN, and RB1, harbored 40 variants of nonsense, missense, and frameshift mutations (Peric et al., 2019). Franz et al., (2017) using genetic sequencing of 50 mutational hotspots, found five mutations in 37 samples of thymoma, HRAS mutation in 3 cases of type A thymoma, and SMARCB1 and STK11 genes in one case of type B3 thymoma. GTF2I is also considered a potential driven gene in thymoma since its correlation with myasthenia gravis is supported (Radovich et al., 2018). In the present study, direct sequencing and sequence analysis of TP53 and EGFR genes were only performed; thus, the primary gene inducing carcinogenesis could not be further investigated in our samples.

Few studies discussed genetic variant among histological spectrum of thymoma. A number of studies investigated other molecular profiles of TP53 and EGFR mutation in terms of thymoma type (Girard et al., 2009, Oberndorfer et al., 2019; Suzuki et al., 2006). Girard et al., reported no p53 and EGFR mutation in thymoma type A, B2, and B3 (Girard et al., 2009). The proportion of p53 mutation was lower in thymoma (thymoma type A, 4,2\%) than thymic carcinoma (21.7\%) according to a previous study (Oberndorfer et al., 2019). A cohort study on forty Chinese patients with thymic epithelial tumor reported no p53 and EGFR mutation in thymoma patients (Wang et al., 2021). This research findings were in line with the abovementioned studies, revealing that the predominant subtype was thymoma type A. EGFR and p53 mutations were frequently spotted in advanced stage of the disease (Sakane, 2021).

In brief, it seems that thymoma type does not affect the genetic alterations. However, further studies on alteration in gene expression profiles of thymoma are needed.

A recent meta-analysis suggested that some biomarkers in thymoma correlated with patients' prognostic (Zeng $\mathrm{H}$, 2020). However, the evidence showing that TP53 and EGFR mutations are the prognostic marker remains inconclusive. However, some past study depicted TP53 
as an indicator of worse prognosis in thymoma. Using International Agency for Research on Cancer (IARC) TP53 database, Victor et al., concluded that TP53 mutation presence could significantly worsen median disease-free survival time (9.72 months vs. 115.05 months, with and without TP53 mutation, respectively) (Victor et al., 2018). This finding suggested that p53 and EGFR could play an essential role in determining patients' prognosis and further urgency to be investigated .

Currently, the cornerstone of thymoma therapy is surgical resection. Moreover, chemotherapy and radiotherapy show promising results for stages III and IV (Detterback et al., 2013). Nevertheless, the role of targeted therapy, especially in TKI in thymoma, is unknown. Even though E709K is a rare mutation known in lung carcinoma (Frega et al., 2016), its detection has potentially become a good candidate for treatment using the $2^{\text {nd }}$ generation TKI, namely, afatinib and neratinib (Kobayashi et al., 2015). Hopefully, the presence of this mutated gene, as displayed in this study, will have the same effect on thymoma patients.

The main limitation of our study was the relatively small sample size. In addition, we did not identify the role of $\mathrm{p} 53$ and EGFR in predicting patients' prognosis.

In conclusion, our study found that the mutational frequency ofexon 6 of p53 and exons 18, 19, and 21 of EGFR genes was low in thymoma. These genes are probably less critical as the primary oncogenic driver in thymoma Indonesian patients. Nevertheless, this study showed two samples with a significant mutation in $p 53$ and $E G F R$ genes, indicating the need for further research on thymoma prognostification and targeted therapy.

\section{Author Contribution Statement}

ES, JZ, DRN conceived, designed and performed the analysis; JZ, RS, RB, DRN, MRF collected the data and did experimental tools; ES, JZ, DRN, MRF wrote the paper. All authors read and approved the final version of this manuscript.

\section{Acknowledgments}

\section{Funding Statement}

The authors would like to thanks Universitas Indonesia for funding this research through PUTI Grant with contract number NKB-4184/UN2.RST/HKP.05.00/2020

\section{Ethical Approval}

This study was reviewed and approved by Persahabatan Hospital Ethic Committee, Ref no : 139/KEPKRSUPP/12/2020.

\section{Availability of data}

The primary data could be obtained upon request and should be approved by Ethical Committee

\section{Conflict of Interest}

Authos affirm no conflict of interest.

\section{References}

Balak MN, Gong Y, Riely GJ, Somwar R, Li AR, Zakowski MF, et al (2006). Novel D761Y and common secondary T790M mutations in epidermal growth factor receptor-mutant lung adenocarcinomas with acquired resistance to kinase inhibitors. Clin Cancer Res, 12, 6494-501.

Bouaoun L, Sonkin D, Ardin M, et al (2016). TP53 variations in human cancers: new lessons from the IARC TP53 database and genomics data. Hum Mutat, 37, 865-76.

De Luca A, Carotenuto A, Rachiglio A, et al (2008) The role of the EGFR signaling in tumor microenvironment. $J$ Cell Physiol, 214, 559-67.

Detterback FC, Zeeshan A (2013). Thymoma: current diagnosis and treatment. Chin Med J, 126, 2186-91.

Frega S, Conte P, Fassan M, Polo V, Pasello G (2016). A triple rare E709K and L833V/H835L EGFR mutation responsive to an Irreversible Pan-HER Inhibitor: A Case Report of Lung Adenocarcinoma Treated with Afatinib. $J$ Thorac Oncol, 11, 63-4.

Giacomelli AO, Yang X, Lintner RE, et al (2018), Mutational processes shape the landscape of TP53 mutations in human cancer. Nat Genet, 50, 1381-7.

Girard N, Shen R, Guo T, et al (2009). Comprehensive genomic analysis reveals clinically relevant molecular distinctions between thymic carcinomas and thymomas. Clin Cancer Res, 15, 6790-9.

Nedooshan JJ, Yazdi MF, Neamatzadeh H, et al (2016). Association between TP53 codon $72 \mathrm{G}>\mathrm{C}$ polymorphism and thyroid carcinoma risk: $\mathrm{An} \mathrm{Up}=$ to-Date Meta-Analysis. Asian Pac J Cancer Biol, 1, 89-95.

Jung H, Lee KS, Choi JK (2021). Comprehensive characterisation of intronic mis-splicing mutations in human cancers. Oncogene, 40, 1347-61.

Kobayashi Y, Togashi Y, Yatabe Y, et al (2015). EGFR Exon 18 mutations in lung cancer: Molecular Predictors of Augmented Sensitivity to Afatinib or Neratinib as Compared with First- or Third Generation TKIs. Clin Cancer Res, 21, 5305-13.

Lee JC, Hung JY, Kim YC, et al (2021). Real-world treatment patterns in patients with EGFR mutation-positive NSCLC receiving a first-line, first- or second-generation EGFR tyrosine kinase inhibitor in South Korea and Taiwan. Asian Pac J Cancer Biol, 6, 123-32.

Lei Y, Ji K, Xin D, Zhen Y, Di G (2019). Genetic characterization of thymoma. Sci Rep, 9, 2369.

Li VD, Li KH, Li JT (2019). TP53 mutations as potential prognostic markers for specific cancers: analysis of data from The Cancer Genome Atlas and the International Agency for Research on Cancer TP53 Database. J Cancer Res Clin Oncol, 145, 625-36.

Lynch TJ, Bell DW, Sordella R, et al (2004). Activating mutations in the epidermal growth factor receptor underlying responsiveness of nonsmall-cell lung cancer to gefitinib. $N$ Engl J Med, 350, 2129-39.

Meister M, Schirmacher P, Dienemann H, et al (2007). Mutational status of the epidermal growth factor receptor (EGFR) gene in thymomas and thymic carcinomas. Cancer Lett, 248, 186-91.

Moreira AL, Won HH, McMillan R, et al (2015). Massively parallel sequencing identifies recurrent mutations in TP53 in thymic carcinoma associated with poor prognosis. J Thorac Oncol, 10, 373-80.

NCCN. National Comprehensive Cancer Network Clinical Practice Guideline in Oncology (NCCN Guidelines): Thymomas and Thymic Carcinoma. 2016.

Oberndorfer F, Mullauer L (2020). Genomic alterations in 
thymoma-molecular pathogenesis?. J Thorac Dis, 12, 7536-44.

Okamoto I, Morita S, Tashiro N, et al (2018). Real world treatment and outcomes in EGFR mutation-positive nonsmall cell lung cancer: Long-term follow-up of a large patient cohort. Lung Cancer, 117, 14-9.

Paz-Ares L, Soulie'res D, Melezınek I, et al (2010). Clinical outcomes in non-small-cell lung cancer patients with EGFR mutations: pooled analysis. J Cell Mol Med, 14, 51-69.

PDPI (2003). Tumor Mediastinum: Pedoman Diagnosis dan Penatalaksanaan di Indonesia. 2003.

Peric J, Samaradzic N, Trifunovic VS, et al (2019). Correlation of genomic alterations and PD-L1 expression in thymoma Arch Med Sci, doi:10.5114/aoms.2020.96537.

Prays J, Ortiz-Villalón C (2021). Molecular landscape of thymic epithelial tumors. Semin Diagn Pathol, S07402570(21)00050-2.

Radovich M, Pickering CR, Felau I, et al (2018). The integrated genomic landscape of thymic epithelial tumors. Cancer Cell, 33, 244-58.

Sadek NA, M Abd-eltawab S, Assem NM, et al (2020). Prognostic value of absolute lymphocyte count, lymphocute percentage, serum albumin, aberrant expression of CD7, CD19, and the tumor suppressors (PTEN and p53) in patients with acute myeloid leukemia. Asian Pac J Cancer Biol, 5, 131-40.

Sakane T, Murase T, Okuda K, et al (2019). A mutation analysis of the EGFR pathway genes, RAS, EGFR, PIK3CA, AKT1 and BRAF, and TP53 gene in thymic carcinoma and thymoma type A/B3. Histopathology, 75, 755-66.

Sakane T, Sakamoto Y, Masaki A, et al (2021). Mutation profile of thymic carcinoma and thymic neuroendocrine tumor by targeted next-generation sequencing. Clin Lung Cancer, 22, 92-9.

Suzuki E, Sasaki H, Kawano O, et al (2006). Expression and mutation statuses of epidermal growth factor receptor in thymic epithelial tumors. Jpn J Clin Oncol, 36, 351-6.

Wang H, Xu X, Luo L, et al (2021). Mutational landscape of thymic epithelial tumors in a Chinese population: insights into potential clinical implications. Gland Surg, 10, 1410-7.

$\mathrm{Xu} \mathrm{F}$, Lin H, He P, et al (2020). A TP53-associated gene signature for prediction of prognosis and therapeutic responses in lung squamous cell carcinoma. Oncoimmunology, 9, 1731943.

Yoh K, Nishiwaki Y, Ishii G, et al (2008). Mutational status of EGFR and KIT in thymoma and thymic carcinoma. Lung Cancer, 62, 316-20.

Zeng H, Yang W, Xu B, et al (2020). Relationship of possible biomarkers with malignancy of thymic tumors: a metaanalysis. BMC Cancer, 20, 928.

Zhan P, Chen X, Wu XY, et al (2018). Mutation analysis of the EGFR gene and its downstream signaling pathway in thymic carcinoma patients from a Chinese Han population. Clin Respir J, 12, 601-7.

\section{అ묘}

This work is licensed under a Creative Commons AttributionNon Commercial 4.0 International License. 